Revista de Antropología Social

ISSNe: 1988-2831

http://dx.doi.org/10.5209/RASO.63776

\title{
\#XCOL: Experimentación para la etnografía contemporánea
}

Estalella, Adolfo y Sánchez Criado, Tomás (Eds.). 2018. Experimental collaborations: Ethnography through fieldwork devices (Vol. 34). Nueva York: Berghahn Books.

Este libro forma parte de una serie de publicaciones producidas desde la European Association of Social Anthropologist (EASA). Fue en el congreso bianual de 2014 de esta misma red donde se establecieron las conversaciones, las condiciones y el impulso necesario para la creación de Experimental collaborations: Ethnography through fieldwork devices, y la posterior red de EASA, \#Colleex - Collaboratory for Ethnographic Experiments. Editado por Adolfo Estalella y Tomás Sánchez Criado, el libro recoge diversos proyectos etnográficos en África, América y Europa que se distanciarían de lo que la antropología clásica ha consolidado como su modo etnográfico de producción de conocimiento: la observación participante. Los distintos capítulos nos van mostrando formas de hacer que se vienen a llamar colaboraciones experimentales. Colaboraciones porque existe un compromiso social con/en el campo y experimentales porque surgen como una especie de desviación de la observación participante, que no pretende desplazarla, pero que transforma las normas y las relaciones en el campo.

En cuanto a la estructura de este monográfico, se inicia con un prefacio escrito por George E. Marcus y finaliza con un epílogo de Sarah Pink donde se incide sobre los aspectos novedosos y los núcleos problemáticos del libro. En la introducción escrita por los editores, Estalella y Sánchez Criado, se recogen aspectos del método etnográfico que debemos problematizar y nos aportan una guía de conceptos como dispositivos, experimentación o colaboraciones experimentales. Estas últimas pueden presentarse según los autores de tres formas distintas:

Modo 1: La colaboración se encuentra integrada en prácticas de trabajo de campo de larga duración que es extractiva y con unas claras relaciones asimétricas.

Modo 2: La forma de abordar el trabajo de campo infundido por un gesto de compromiso político o ético donde la colaboración se enmarca, en estas circunstancias, como una estrategia para articular la responsabilidad ética y los compromisos políticos de los antropólogos en el campo, hasta el punto que, en algunos casos, obliga a la antropóloga a sacrificar sus objetivos de investigación por los de sus homólogos y a adaptar el trabajo de campo a las situaciones que ellos podrían predefinir.

Modo 3: Define el espacio conceptual y práctico para concebir la colaboración como innovación en el método, una experimentación para intentar crear una forma diferente de trabajo de campo que se comprometa metodológicamente en exploraciones epistémicas conjuntas.

A pesar de esta categorización, como podemos ver a lo largo de los ocho capítulos, todas son opciones variables que se prestan a la transformación. No obstante, es el Modo 3 la que considero que merece más atención y que aporta otras formas de hacer y pensar la etnografía. Así, el Modo 3 nos aproxima a la experimentación etnográfica donde el etnógrafo, junto a sus homólogos, se adentra en la creación de 
espacios de producción/creación de conocimiento a través de procesos e intervenciones materiales y sociales que convierten el campo en un sitio de colaboración epistémica, un sitio para la construcción y la problematización conjunta. Por tanto, la experimentación nos abre la puerta a las imaginaciones etnográficas, transformando a los informantes de campo en compañeros epistémicos y buscando formas de remediar la forma en la que la etnografía se acompaña de los otros.

Haciendo un breve recorrido por los capítulos de este libro, nos encontramos en primer lugar con el capítulo escrito por Emma Garnett, "Experimenting with Data: 'Collaboration' as Method and Practice in an Interdisciplinary Public Health Project”. En este capítulo, la autora explora las relaciones y prácticas colaborativas en un proyecto multidisciplinario de salud pública donde se centra en abordar los desafíos de la colaboración que enfrentan los científicos del proyecto y ella misma como antropólogo. Así, reflexiona sobre algunas de las formas en que los roles de científico/colaborador, antropólogo/colaborador fueron negociados y desarrollados a lo largo del proyecto.

En el segundo capítulo, “The 'Research Traineeship': The Ups and Downs of Parasiting Ethnography”, Maria Schiller reflexiona sobre el impacto de la colaboración en su investigación y nos sugiere algunas consideraciones que plantearnos para la realización de etnografías colaborativas en organizaciones estatales. En el tercer capítulo, "Finding One's Rhythm: A 'Tour de Force' of Fieldwork on the Road with a Band", Anna Lisa Ramella nos lleva en movimiento y nos sumerge en ritmos de una gira. Su propia experiencia móvil y rítmica le llevan a la necesidad de buscar métodos de campo más creativos, sensoriales e intuitivos. De forma muy interesante Ramella utiliza un vocabulario que nos habla de ritmos y movilidad para sumergirnos en su narración de prácticas de trabajo de campo.

Andrea Gaspar en el cuarto capítulo, "Idiotic Encounters: Experimenting with Collaborations between Ethnography and Design", nos sumerge en un compromiso con las prácticas del diseño especulativo. Gaspar se inspira en la figura del idiota de Stengers que Mike Michael problematiza, proponiendo el potencial de los objetos idiotas para la investigación social y la idiotez proactiva como una forma de interrogarnos acerca de lo que estamos haciendo como investigadores sociales (Michael 2012a, 2012b). De esto modo, la figura del idiota llevada al campo de la etnografía abre la posibilidad de forjar otras relaciones con otras formas de saber. Además, Gaspar hace un interesante ejercicio recogiendo las múltiples fricciones que surgieron en su trabajo de campo. Estas fricciones como dispositivo de participación en el trabajo nos hacen ser conscientes de que se requiere ser creativo en términos de diseño, pero que además estamos expuestos a los efectos transformadores de nuestras prácticas de campo.

De las investigaciones incluidas en este volumen, el que más se alinea con mi interés actual es el quinto capítulo, "Fieldwork as Interface: Digital Technologies, Moral Worlds and Zones of Encounter”, de Karen Waltorp. En este artículo Karen llama la atención sobre cómo la tecnología digital permite la formación interactiva del conocimiento y la retroalimentación continua de manera específica. También discute cómo la creación de conocimiento en su proyecto ha integrado la forma en que los interlocutores y ella misma utilizan los medios sociales. Recientemente junto a Marta Morgade Salgado, en una investigación que comparte ciertos puntos con la de Waltorp, documentamos la vida cotidiana de los jóvenes inmigrantes institucionalizados en centros de menores y su uso de los medios digitales, y cómo la inclusión de estos medios digitales supuso una transformación en nuestras prácticas de campo (Mendoza y Morgade, en prensa). 
En el sexto capítulo, "Thrown into Collaboration: An Ethnography of Transcript Authorization", Alexandra Kasatkina, Zinaida Vasilyeva y Roman Khandozhko nos presentan una pieza colaborativa que recoge una investigación basada en entrevistas que buscan documentar la vida y las carreras de los científicos involucrados en el proyecto nuclear Obninsk. Este trabajo nos hace reflexionar sobre cómo el propio proceso de autorización crea un nuevo espacio comunicativo incierto, donde todos los participantes se encuentran constantemente con la necesidad de improvisar y comprometerse. Así, veremos cómo transitar por ese nuevo espacio incógnito y en constante cambio exige esfuerzos mutuos significativos.

El séptimo capítulo, "A Cultural Cyclotron: Ethnography, Art Experiments, and a Challenge of Moving towards the Collaborative in Rural Poland", de Tomasz Rakowski centra la práctica etnográfica entendida como una hibridación entre la acción social y artística. En detalle nos muestra cómo introducir elementos del arte en la etnografía procurando no establecer relaciones de dominación, desigualdad simbólica y cultural en regiones rurales y desfavorecidas de Polonia. Este interesante capítulo nos hace reflexionar sobre cómo las prácticas relacionadas con el arte pueden transformar lo que entendemos por arte y etnografía.

En el último trabajo, "Making Fieldwork Public: Repurposing Ethnography as a Hosting Platform in Hackney Wick, London", Isaac Marrero-Guillamón nos da cuenta de un inesperado devenir colaborativo que se aleja de lo que debía haber sido un ejercicio clásico de trabajo de campo. Sumergido en una trayectoria inesperada en estrecha colaboración con artistas, nos plantea que una reimaginación exitosa de la etnografía requiere del esfuerzo colectivo para encontrar formas de reconocer, asimilar y transmitir las formas de conocimiento concomitantes a los foros a los que apelamos.

Todos los capítulos que componen esta obra resultan de gran utilidad, especialmente, para aquellos investigadores que deseen o necesiten, por las características de su objeto de estudio, encontrar nuevas formas de relacionarse con la vida cotidiana. Así, este libro es una invitación a jugar con la etnografía para hacerla más interesante para los investigadores y los participantes. Podríamos decir que la etnografía no deja de ser un proceso de aprendizaje incierto tanto para los participantes como para los investigadores quienes están probando mejores formas de relacionarse. Como hemos visto esto no es tarea fácil y el etnógrafo deberá caracterizarse por su apertura y su capacidad para adaptarse a los acontecimientos inesperados.

De este modo, la investigación siempre tendrá un carácter performativo y será una práctica desordenada y frágil que se mezcla con los fenómenos que estudia. El reto de la experimentación no es tomar la improvisación o lo incierto como un fracaso metodológico, sino como una inspiración para pensar el método de una manera más inclusiva. Recordamos que lo experimental también señala la ética, la colaboración, que adapta constantemente las relaciones de investigación a fin de maximizar las posibilidades de que los investigadores y los participantes se comprendan un poco mejor. Por lo tanto, ese Modo 3 es un intento por evitar el pensamiento reduccionista, donde la desestabilización del método nos abre un mundo de encuentro productivo con los cuerpos, espacios, hechos, sucesos.

Karmele Mendoza Pérez Dpto. Psicología y Antropología Universidad de Extremadura karmelemp@unex.es 


\section{Bibliografía}

Mendoza Pérez, Karmele; Morgade Salgado, Marta (en prensa). "Mobility and the Mobile: A Study of Adolescent migrants and their use of the mobilephone". Mobile Media \& Communication.

Michael, Mike (2012a). "De-signing the Object of Sociology: Toward an "Idiotic" Methodology". The Sociological Review, 60 (S1): 166-83.

- (2012b). "What Are We Busy Doing?: Engaging the Idiot". Science, Technology, \& Human Values, 37 (5): 528-54. 